\title{
Next-generation crop engineering
}

\author{
The rapid development of plant biotechnologies is profoundly shaping crop breeding and catalysing the next \\ revolution in agriculture.
}

T: he basic principle of crop breeding is to first discover and then select for variants with desired traits. While selection is relatively easy, discovery is more challenging. Conventional breeding for domestication and crop improvement have unquestionably revolutionized agriculture and our society. But to further explore the potential of agriculture to feed an ever-growing population, larger crop diversity needs to be unlocked. The gene editing and RNA viral transfection technologies developed over recent years allow precise engineering of desirable variants with unprecedentedly high efficiency and resolution, greatly expanding the range of variations available and reducing our reliance on naturally existing mutations.

Most agronomic traits are quantitative, and controlled by multiple genes. In conventional breeding, their improvement needs multiple rounds of crossing and gene pyramiding, which is laborious and time consuming. The efficacy relies on the available diversity and is affected by linkage drag of deleterious mutations. Mutation breeding involves exposing seeds to chemicals or radiation, which is a less targeted approach as mutations occur randomly throughout the genome. Most mutations cause severe phenotypic defects and are thus unusable for practical purposes, making the tracking of a rare beneficial mutation extremely difficult.

Due to the low accessibility of desirable variations or gene combinations, cross- and mutation breeding largely depend on luck. This breeding philosophy was broken by transgenic breeding, which deliberately introduces foreign genes of known function into a crop to increase yield, resistance or nutritional value. This strategy enables breeding by design, but its products, especially genetically modified organisms (GMOs) as food crops, are subjected to strict regulation. Consequently, only a small number of GMOs are commercialized in certain countries.

In 2017, Zachary Lippman's laboratory targeted the promoters of developmental genes using CRISPR-Cas editing, generating multiple promoter-edited alleles in tomato and creating a continuum of phenotypic variation of yield-related traits $^{1,2}$. MADS-box genes and genes in the CLAVATA-WUSCHEL circuit were selected, because they control important yield traits and are dose-sensitive. In the current issue of Nature Plants, David Jackson's group apply a similar strategy to maize by engineering three $C L V$ genes ${ }^{3}$. Weak promoter-edited alleles and null alleles were generated, conferring quantitative variations of grain yields in the edited lines, some of which show impressive field performance.

This work shows that quantitative trait variations can be successfully engineered by editing developmental regulatory genes. Although phenotypic effects cannot be predicted from allele type or transcriptional change (because of non-linear relationships between transcriptional and phenotypic changes ${ }^{2}$ ), subtle phenotypic manipulation can be achieved, and favourable variants can be obtained from the continuum of trait variations created.

Besides crop improvement, CRISPR-Cas technology can be used for de novo domestication or re-domestication. This uses a knowledge of domestication genes and the fact that orthologous genes in closely related species control the same or related phenotypes. Editing domestication genes or their homologues has been done in wild tomato ${ }^{4,5}$, ground cherry ${ }^{6}$ and tetraploid rice $^{7}$, which successfully transformed the wild species to cultivar-like plants.

CRISPR-Cas breeding is more efficient than mutation breeding because mutagenesis is targeted to genes known to control desirable traits. Moreover, transgene-free plants can be easily obtained by transiently expressing CRISPR proteins or by segregating out constitutively expressed CRISPR. Gene-edited crops could thus avoid regulations against the cultivation of GMOs. A few countries have already approved crops derived from certain types of genome editing ${ }^{8}$. For example, the United States Department of Agriculture has approved over 70 gene-edited crops ${ }^{9}$, and a gene-edited tomato containing higher contents of gamma-aminobutyric acid was recently approved in Japan ${ }^{10}$.

To unlock high levels of variation by gene editing, at least three things are currently missing. A better understanding is needed of key regulators for evolutionarily or developmentally important genes. It will also be critical to dissect gene networks controlling target phenotypes and cis-regulatory networks affecting gene expression. Finally, for most species, stable and efficient transformation and regeneration procedures must be established. Unless in planta gene editing is developed quickly, gene-editing breeding will be unable to benefit such recalcitrant species.

Alternative strategies for next-generation crop engineering exist. RNA viral transfection is a spray-on technology enabling transient fine-tuning of agronomic traits, as implemented in multiple species reported by Torti et al. last month ${ }^{11}$. The researchers transiently expressed or silenced regulatory genes in multiple hormonal circuits to modulate traits, such as flowering time, height and stress response in species including Arabidopsis, Nicotiania benthamiana, tomato, rice, Brassica napus and soybean. As the RNA virus is not transmissible across generations, this approach allows for temporary plastic manipulations of traits without any modification of genetic material.

Crop breeding need no longer rely on naturally occurring mutations, but instead artificially generated variations can be the raw material for further breeding. A much broader spectrum of phenotype space is ready for exploration, allowing development of optimal phenotypes adapted to heterogeneous environments on Earth, or even space. A new biotechnology-driven revolution in agriculture could be just around the corner.

Published online: 17 March 2021 https://doi.org/10.1038/s41477-021-00890-5

References

1. Soyk, S. et al. Cell 169, 1142-1155 (2017).

2. Rodríguez-Leal, D. et al. Cell 171, 470-480 (2017).

3. Liu, L. et al. Nat. Plants https://doi.org/10.1038/s41477-02100858-5 (2021).

4. Zsögön, A. et al. Nat. Biotechnol. 36, 1211-1216 (2018).

5. Li, T. et al. Nat. Biotechnol. 36, 1160-1163 (2018).

6. Lemmon, Z. H. et al. Nat. Plants 4, 766-770 (2018).

7. Yu, H. et al. Cell https://doi.org/10.1016/j.cell.2021.01.013 (2021).

8. Menz, J. et al. Front. Plant Sci. 11, 586027 (2020).

9. Bomgardner, M. Gene-edited crops approved by USDA jumps from 7 to 70 between 2019 and 2020. Genetic Literacy Project http://go.nature.com/2ZREgvN (2020).

10. Kojima, M. Success of CRISPR tomato may determine if gene-edited foods take root in Japan. Genetic Literacy Project http://go.nature.com/3aY65Zz (2021).

11. Torti, S. et al. Nat. Plants 7, 159-171 (2021). 